\title{
Congenital Hypothyroidism Presenting with Seizures and Pseudo-Hirschsprung's Disease in Newborn
}

\author{
Deepak Sharma $\cdot$ Srinivas Murki $\cdot$ G. Dhanraj \\ Received: 31 July 2013 / Accepted: 16 October 2013 /Published online: 6 November 2013 \\ (C) Dr. K C Chaudhuri Foundation 2013
}

\begin{tabular}{|ll|}
\hline \multicolumn{2}{|l|}{ Abbreviations } \\
CH & Congenital Hypothyroidism \\
HD & Hirschsprung's Disease \\
TSH & Thyroid Stimulating Hormone \\
USG & Ultrasonogram \\
\hline
\end{tabular}

To the Editor: Congenital Hypothyroidism $(\mathrm{CH})$ is one of the most common preventable causes of mental retardation. Thyroid screening is done in an infant with post maturity, macrosomia, wide open posterior fontanel, prolonged unconjugate jaundice, constipation, poor feeding, hypotonia, hoarse cry, umbilical hernia, macroglossia and dry or edematous skin and in a child with growth delay and thyroid swelling [1]. In this report, we describe a rare association of seizures and pseudo Hirschsprung's disease with hypothyroidism in a neonate.

A male newborn, $3 \mathrm{~kg}$ at birth presented on day 4 of life with abdominal distension and bilious vomiting. On abdominal examination, there was divarication of recti, prominent veins; visible bowel loops and bowel sounds were present on auscultation. C-reactive protein was negative, serum electrolytes, kidney and liver function tests were normal. Plain abdominal radiograph and USG abdomen revealed dilated gas filled bowel loops. Barium enema revealed faint opacification of rectum and descending colon with air distended proximal colon. At the 2nd hour of admission, the infant was noted to have recurrent seizures that got controlled with Phenobarbital. Seizure workup was normal. As the infant had persistent unexplained abdominal distension, thyroid function tests done on day 4 showed low T3

\footnotetext{
D. Sharma $\cdot$ S. Murki $(\bowtie)$

Department of Pediatrics, Fernandez Hospital, Opposite Old MLA quarters, Hyderabad 500029, Andhra Pradesh, India e-mail: srinivasmurki2001@gmail.com

G. Dhanraj

Department of Pediatrics, Paramitha Children's Hospital, Hyderabad, Andhra Pradesh, India
}

$(0.10 \mathrm{ng} / \mathrm{mL})$, low T4 $(0.8 \mu \mathrm{g} / \mathrm{dL})$ and high Thyroid Stimulating Hormone (TSH $>150 \mu \mathrm{IU} / \mathrm{mL}$ ). Maternal thyroid profile was normal. Baby responded well to thyroxin started at $15 \mu \mathrm{g} / \mathrm{kg} / \mathrm{day}$ on the 6th day. Thyroid nuclear scan showed non visualization of thyroid gland consistent with congenital Athyrogenesis.

Persistent clinical features with sluggish bowel motility on the ultrasound, abnormal thyroid profile, and good clinical response to oral thyroxin are suggestive of an association of congenital hypothyroidism with pseudo intestinal obstruction [2] Transient neonatal seizures have previously been reported to be associated with congenital hypothyroidism [3]. The likelihood of an accidental association between hypothyroidism and seizures in this infant was low as metabolic parameters, sepsis screen, blood cultures and neuroimaging were all normal. The purpose of this case report is to encourage pediatrician to investigate for hypothyroidism in clinically suspected pseudo intestinal obstruction and as a routine screening in all sick newborns.

Contributions SM: Conceptualized the case report, reviewed and revised the manuscript and approved the final manuscript as submitted. DS: Drafted the initial manuscript and approved the final manuscript as submitted. GD: Critically reviewed the manuscript, and approved the final manuscript as submitted. Dr. Srinivas Murki will act as guarantor for this paper.

Conflict of Interest None.

Role of Funding Source None.

\section{References}

1. Seth A, Aggarwal V, Maheshwari A. Hypothyroidism in children beyond $5 \mathrm{y}$ of age: Delayed diagnosis of congenital hypothyroidism. Indian J Pediatr. 2012;79:891-5.

2. Al Jurayyan NAM, Al Asmi MM, Al Jurayyan RNA. Transient functional intestinal obstruction in a newborn: The first clinical manifestation of congenital hypothyroidism. Curr Pediatr Res. 2011;15:101-3.

3. Sutsko RP, Braziuniene I, Saslow JG, Razi N, Amendolia B, Stahl G, et al. Intractable neonatal seizures: An unusual presentation of congenital hypothyroidism. J Pediatr Endocrinol Metab. 2009;22: 961-3. 